\title{
MISSIONS ASIATIQUES
}

Léon Bourdon, La Compagnie de Jésus et le Japon (1547-1570). Lisbonne/Paris, Centre culturel portugais de la fondation Calouste Gulbenkian/Commission nationale pour la commémoration des découvertes portugaises, 1993. $20 \times 28$, 723 p., bibliogr., index, cartes.

Les Portugais au Tibet. Les premières relations jésuites (1624-1635). Trad. du portugais et éd. par Hugues Didier. Paris, Chandeigne, 1996. $15 \times 22,382$ p., bibliogr., index, cartes (Magellane).

Jean-Pierre DuteIL, L'Ombre des nuages. Histoire et civilisation du Vietnam au temps des Là et au début de la dynastie Nguyàn (1427-1819). Paris, Arguments, 1997. $17 \times 25$, II-186 p., bibliogr., index, cartes.

Jacques Proust, La Supercherie dévoilée. Une réfutation du catholicisme au Japon au xvif siècle écrite par Cristóvão Ferreira. Texte trad. par Jacques et Marianne Proust. Paris, Chandeigne, 1998. $15 \times 22$, 192 p., bibliogr., « petit dictionnaire " (Magellane).

L'histoire des missions en Asie a décidément pris l'habit de l'histoire culturelle. Ces cinq ouvrages, bien qu'ils couvrent des domaines géographiques fort différents, le Japon, le Tibet et le Vietnam, du xvi ${ }^{e}$ au xviI siècle, témoignent d'un goût prononcé de leurs auteurs pour une histoire davantage centrée sur les hommes et les missionnaires que sur les intentions de leurs supérieurs hiérarchiques, laïcs ou ecclésiastiques.

Cela étant, la publication de la thèse de Léon Bourdon, La Compagnie de Jésus et le Japon (1547-1570), présentée en 1951 pour le doctorat ès lettres, nous fait mesurer la distance parcourue depuis les années 1950 , et ceci compte tenu du fait que l'histoire des missions ne fait pas partie des champs de la recherche les plus enclins aux effets de mode. L'ouvrage est empreint du désir d'écrire une histoire générale des missions jésuites au Japon. En témoigne le large usage que fait son auteur d'une histoire du Japon, celle de Luis Fróis, écrite au milieu du Xvi ${ }^{\mathrm{e}}$ siècle, et qui donne à sa thèse sa continuité narrative. Or ce désir d'une histoire globalisante renvoie à celui des divers supérieurs et historiographes de la Compagnie de Jésus. Est-il besoin de rappeler, en effet, que la Compagnie a consacré, pratiquement dès sa création, une partie de son énergie à écrire l'histoire des missions en même temps

Revue de synthèse : $4^{\mathrm{c}} \mathrm{S}$. $\mathrm{n}^{\text {os }} 2-3$, avr.-sept. 1999, p. 482-485. 
qu'elle était vécue par divers acteurs? Cette intention fondatrice a laissé, à notre avis, une marque indélébile dans l'écriture de l'histoire des missions au $x^{e}$ siècle. Ceci, par ailleurs, n'est ni surprenant ni original si l'on considère qu'une partie de l'histoire des souverains de France ou de la péninsule ibérique est produite selon le même schéma : une historiographie officielle écrite aux époques anciennes et chargée de justifier l'action des souverains, et une histoire écrite au xxx siècle, qui reprend, en y introduisant une distance plus ou moins grande, l'histoire des grands hommes.

L'histoire écrite par Bourdon passe ainsi en revue les hommes importants dans le développement de la mission du Japon : François Xavier, Jorge Alvares, Cosme de Torres... De cette histoire disparaissent quelque peu les hommes de second plan et les subalternes tandis que, du côté japonais, le récit est partagé entre le portrait assez précis de quelques grands convertis ou informateurs japonais (le marchand Yajirô, le lettré zen « Paulo de Funai ») et l'évocation d'une multitude anonyme d'autres convertis dont l'identité reste difficile à cerner. Il reste de cette étude divers thèmes proposés à la sagacité de futurs chercheurs : l'inscription de la mission dans le contexte des troubles politiques de la première moitié du xvi siècle, la question de l'adaptation du christianisme aux religions japonaises, les problèmes relatifs à la transcription des termes chrétiens et les controverses entre les religieux japonais et les missionnaires... Ce livre ouvre donc la voie, d'une part, à des études plus larges, d'autre part, à des approches qui relèvent davantage de la micro-histoire.

Dans la première veine s'inscrit le livre de Jean-Pierre Duteil, L'Ombre des nuages, qui n'est pas, à proprement parler, un livre d'histoire des missions. Il replace au contraire les missions catholiques dans le contexte de la "civilisation" vietnamienne en utilisant des sources diverses : mémoires historiques rédigés sous différentes dynasties, lettres et journaux conservés aux Archives des Missions étrangères de Paris, relations imprimées et histoires anciennes... Détournant certaines de ces sources de leur raison d'être première, l'auteur suggère que les archives missionnaires puissent servir à autre chose qu'à une simple histoire des missions et bien plutôt à une enquête sur l'identité vietnamienne, enquête qu'il mène sur le temps long, en recherchant les diverses influences culturelles qui donnent à cette partie du monde sa cohérence. Les pages qu'il consacre à l'histoire des missions rappellent la place qu'occupaient les jésuites et les Missions étrangères de Paris mais également le rôle exceptionnel que jouèrent bon nombre de catéchistes vietnamiens dans le soutien aux chrétientés indigènes.

L'histoire des missions est donc aussi une histoire des réalités du terrain. À ce titre, les ouvrages de Jacques Proust et de Hugues Didier participent, chacun à leur façon, d'une approche micro-historique. S'il est inévitable que l'histoire s'accroche aux hommes de quelque importance qui ont laissé des têmoignages précieux de leurs parcours, la récente édition par Didier de récits de jésuites portugais au Tibet invite à réfléchir sur la lecture historique d'un type de sources. D'abord, il est clair que les lettres des PP. António de Andrade, Francisco de Azevedo, Estêvão Carcela et João Cabral constituent un ensemble à partir duquel s'élaborera une première connaissance sur le Tibet, son climat, son agriculture, sa religion et ses mœurs. Cependant, cette dernière n'a rien d'une approche anthropologique : les jésuites sont 
dans l'impossibilité de concevoir l'étrangeté culturelle et tentent de rapporter l'inconnu au déjà connu. Dans certains cas, d'ailleurs, cette approche fait rejouer des courants prophétiques plus anciens : ainsi les traits caractéristiques du bouddhisme, le récit de la vie de Bouddha, certains aspects de la vie monastique, leur semblent être - et l'effet de miroir est fort - des reflets déformés d'une conversion ancienne du Tibet au christianisme. Ensuite, l'intention qui préside à la rédaction de ces lettres et l'identité de leurs divers destinataires invitent à s'interroger sur les rapports qu'entretiennent récit et expérience vécue. Ainsi, dans le cas du P. de Andrade, sa relation est quelque peu différente selon qu'il écrit à son roi ou à son supérieur à Rome. En fait, le statut de ces missionnaires jésuites est complexe car ces derniers relèvent à la fois du patronage royal portugais et de l'autorité centrale de leur ordre. Par conséquent, encourager le roi à soutenir les missions d'un côté et, d'un autre côté, montrer son mérite sans oublier le devoir d'obéissance tient parfois de la gageure. Enfin, si le mot « missionnaire " provient de "missus » (envoyé), les parcours de ces jésuites portugais donnent plutôt le sentiment d'une relative autonomie de ces derniers vis-à-vis du pouvoir temporel en Europe. Il ne pouvait guère en être autrement vu l'éloignement de la mission du Tibet par rapport aux centres de décisions et d'information que sont Rome mais également Goa qui joue le rôle de «pôle régional » des missions. De plus, l'autonomie des jésuites se comprend dans un contexte où les décisions devaient parfois être prises très rapidement. La richesse de ces récits, dont une partie sera lue à travers l'Europe érudite, et l'édition rigoureuse qui en est proposée ici ne peut qu'inviter l'historien à un retour aux textes.

Le second ouvrage, La Supercherie dévoilée de Proust, pourrait lui aussi être qualifié d'approche micro-historique. Son propos, annoncé dans le précédent livie du même auteur (L'Europe au prisme du Japon. xvi'-xvII' siècle, Paris, Albin Michel, 1997; cf. le compte rendu d'Isabelle Brian dans la Revue de synthèse, t. CXVIII, 4, 1997, p. 583-586), consiste à redonner sa juste place à une réfutation du catholicisme écrite en 1636, au Japon, par un certain Cristóvão Ferreira. Signé de deux noms, Cristóvão Ferreira et du prénom Chûan, ce texte a constitué longtemps une énigme pour les historiens : fallait-il l'attribuer à quelque philosophe japonais néoconfucianiste ou était-il pensable, compte tenu de sa construction et de sa rédaction, de l'imputer à Ferreira? Le dilemme provient essentiellement du fait que la trajectoire de son auteur est troublante. Proust brosse un portrait du jésuite Ferreira depuis son entrée dans la Compagnie en 1596 jusqu'à son supplice et son abjuration en 1633, en passant par ses années d'apprentissage à Macao, puis au séminaire d'Arima au Japon, son séjour à Miyako entre 1612 et 1617, puis à Nagasaki. Exerçant tour à tour la charge de secrétaire, de trésorier, puis de provincial de facto, cet homme qui fait partie de l'élite de la Compagnie est à la fois un homme de l'administration des missions et un homme de terrain. Après son abjuration, Ferreira intègre la société japonaise, se marie, se fait immatriculer dans un temple zen, et, pensionné par l'État, sert d'agent de renseignement à l'inquisition japonaise.

Cet itinéraire inhabituel conduit le personnage à devenir un homme de plume après 1633 et, comme le suggère Proust, c'est l'œuvre de Ferreira-Chûan qui permet de comprendre l'homme et non l'inverse. L'enquête se penche sur la construction du texte de Ferreira et, à la faveur d'un important appareil critique, démontre qu'elle est non pas le produit d'une imagination japonaise mais le résultat d'une solide for- 
mation jésuite, d'une pratique de la rhétorique et d'un entraînement à l'exercice de la dispute. De façon convaincante, l'analyse montre comment ce texte en apparence simpliste emprunte ses arguments à la Bible, à Aristote, à Thomas d'Aquin, aux conciles, aux disputes qui, au Moyen Âge, opposèrent vérités de la raison et vérités de la foi et même à Érasme. Le texte de Ferreira, s'il est pétri de culture jésuite, donne le vertige : on y voit le long processus d'acculturation jésuite, saupoudré de quelques connaissances sur la philosophie chinoise, déboucher, certes à l'échelle d'un seul individu, sur une réfutation en règle de la doctrine chrétienne. On y voit également un homme, dont le panorama intellectuel est riche, et qui, dans une situation de contrainte, s'appuie sur sa formation passée pour argumenter un renversement radical de ses positions.

Ainsi, l'histoire des missions ne fonctionne plus en vase clos. Elle tient désormais compte de l'implication des jésuites dans les sociétés coloniales. Enfin, une histoire davantage centrée sur l'étude des textes s'interroge sur les modes de transmission culturelle, la logique des échanges, et cela en accordant une place nouvelle à l'analyse de parcours individuels.

Pascale GiRARD (janvier 1999). 